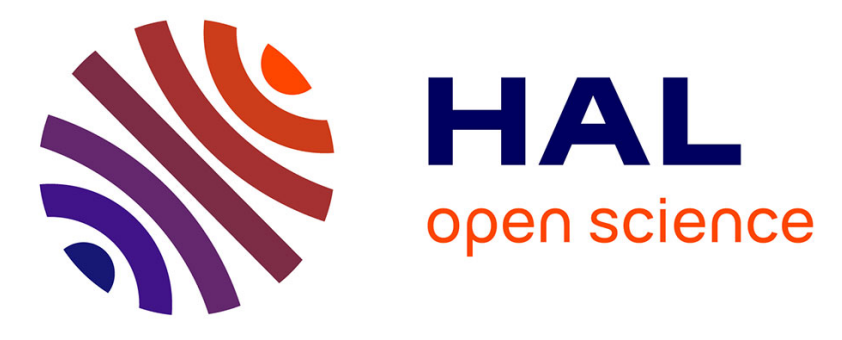

\title{
Four-Year Follow-up of Diagnostic Service in USH1 Patients
}

Anne-Françoise Roux, Valérie Faugère, Christel Vache, David Baux, Thomas

Besnard, Susana Léonard, Catherine Blanchet, Christian Hamel, Michel

Mondain, Brigitte Gilbert-Dussardier, et al.

\section{To cite this version:}

Anne-Françoise Roux, Valérie Faugère, Christel Vache, David Baux, Thomas Besnard, et al.. FourYear Follow-up of Diagnostic Service in USH1 Patients. Investigative Ophthalmology \& Visual Science, 2011, 52 (7), pp.4063-4071. 10.1167/iovs.10-6869 . hal-02444128

\section{HAL Id: hal-02444128 \\ https://hal.umontpellier.fr/hal-02444128}

Submitted on 5 Feb 2020

HAL is a multi-disciplinary open access archive for the deposit and dissemination of scientific research documents, whether they are published or not. The documents may come from teaching and research institutions in France or abroad, or from public or private research centers.
L'archive ouverte pluridisciplinaire HAL, est destinée au dépôt et à la diffusion de documents scientifiques de niveau recherche, publiés ou non, émanant des établissements d'enseignement et de recherche français ou étrangers, des laboratoires publics ou privés.

\section{(1) (1) $\$$}

Distributed under a Creative Commons Attribution - NonCommercial - NoDerivatives 44.0 


\title{
Four-Year Follow-up of Diagnostic Service in USH1 Patients
}

\author{
Anne-Françoise Roux, ${ }^{1,2}$ Valérie Faugère, ${ }^{1}$ Christel Vaché, ${ }^{1}$ David Baux, ${ }^{1}$ \\ Thomas Besnard, ${ }^{1,2,3}$ Susana Léonard, ${ }^{1}$ Catherine Blanchet,${ }^{4}$ Christian Hamel, ${ }^{4}$ \\ Michel Mondain, ${ }^{4}$ Brigitte Gilbert-Dussardier, ${ }^{5}$ Patrick Edery, ${ }^{6,7}$ Didier Lacombe, ${ }^{8}$ \\ Dominique Bonneau, ${ }^{9,10}$ Muriel Holder-Espinasse, ${ }^{11}$ Umberto Ambrosetti, ${ }^{12}$ \\ Hubert Journel, ${ }^{13}$ Albert David, ${ }^{14}$ Geneviève Lina-Granade, ${ }^{15}$ Sue Malcolm, ${ }^{16}$ \\ and Mireille Claustres ${ }^{1,2,3}$
}

\begin{abstract}
Purpose. The purpose of this study was to establish the mutation spectrum of an Usher type I cohort of 61 patients from France and to describe a diagnostic strategy, including a strategy for estimating the pathogenicity of sequence changes.

Methods. To optimize the identification of Usher (USH)-causative mutations, taking into account the genetic heterogeneity, preliminary haplotyping at the five USH1 loci was performed to prioritize the gene to be sequenced, as previously described. Coding exons and flanking intronic sequences were sequenced and, where necessary, semiquantitative PCR and multiplex ligation-dependent probe amplification (MLPA) were performed to detect large genomic rearrangements.
\end{abstract}

From the ${ }^{1}$ Laboratoire de Génétique Moléculaire and the ${ }^{4}$ Centre National de Référence Maladies Rares Affections Sensorielles Génétiques, CHU (Centre Hospitalier Universitaire), Montpellier, Montpellier, France; ${ }^{2}$ INSERM (Institut National de la Santé et de la Recherche Médicale), Unité 827, Montpellier, France; ${ }^{3}$ University Montpellier I, Montpellier, France; ${ }^{5}$ Service de Génétique Médicale, CHU de Poitiers, Poitiers, France; ${ }^{6}$ Service de Cytogénétique Constitutionnelle, Hospices Civils de Lyon, Bron, France; ${ }^{7}$ Unité EA 4171, Université de Lyon, Lyon, France; ${ }^{8}$ Génétiques Médicale, CHU de Bordeaux, Université de Bordeaux 2, Bordeaux, France; 'Service de Génétique, CHU Angers, Angers, France; ${ }^{10}$ UMR (Unité Mixte de Recherche) CNRS 6214 (Centre National de la Recherche Scientifique)/INSERM 771, Angers, France; ${ }^{11}$ Service de Génétique Clinique, CHU de Lille, Lille, France; ${ }^{12}$ Dipartimento di Scienze Chirurgico Specialistiche, Università degli Studi di Milano, UOC (Unità Operativa Complessa) di Audiologia, Fondazione IRCCS (Istituto Ricerca e Cura a Carattere Scientifico) Cà Granda, Ospedale Maggiore Policlinico, Milano. Italy; ${ }^{13}$ Génétique Médicale, CH Bretagne-Atlantique, Vannes, France; ${ }^{14}$ Service de Génétique Médicale, CHU de Nantes, Nantes, France; ${ }^{15}$ Service d'ORL (OtoRhino-Laryngologie), CHU Edouard Herriot, Lyon, France; and ${ }^{16} \mathrm{Clini}-$ cal and Molecular Genetics, Institute of Child Health, London, United Kingdom.

Supported in part by le Ministère de la Recherche "PHRC (Le Programme Hospitalier de Recherche Clinique) National 2004, PROM 7802," AG2R Foundation, and SOS Rétinite.

Submitted for publication November 10, 2010; revised February 2 , 2011; accepted February 23, 2011.

Disclosure: A.-F. Roux, None; V. Faugère, None; C. Vaché, None; D. Baux, None; T. Besnard, None; S. Léonard, None; C. Blanchet, None; C. Hamel, None; M. Mondain, None; B. GilbertDussardier, None; P. Edery, None; D. Lacombe, None; D. Bonneau, None; M. Holder-Espinasse, None; U. Ambrosetti, None; H. Journel, None; A. David, None; G. Lina-Granade, None; S. Malcolm, None; $\mathbf{M}$ Claustres, None

Corresponding author: Anne-Françoise Roux, Laboratoire de Génétique Moléculaire, CHU Montpellier, IURC, 641 Avenue du Doyen Gaston Giraud, F-34093 Montpellier cedex 5, France;

anne-francoise.roux@inserm.fr.
Results. Four years' experience confirms that the chosen approach provides an efficient diagnostic service. Sixty-one patients showed an abnormal genotype in one of the five USH1 genes. Genetic heterogeneity was confirmed, and, although $M Y O 7 A$ remains the major gene, involvement of other genes is considerable. Distribution of missense, splicing, premature termination codons (PTCs; due to point substitution and small deletions/ or insertions), and large genomic alterations was determined among the USH genes and clearly highlights the need to pay special attention to the diagnostic approach and interpretation, depending on the mutated gene.

Conclusions. Over the 4 years of a diagnostic service offering USH1 patient testing, pathogenic genotypes were identified in most cases ( $>90 \%)$. The complexity and heterogeneity of mutations reinforces the need for a comprehensive approach. Because $32 \%$ of the mutations are newly described, the results show that a screening strategy based on known mutations would have solved less than $55 \%$ of the cases. (Invest Opbthalmol Vis Sci. 2011;52:4063-4071) DOI:10.1167/iovs.10-6869

$\mathrm{U}$ sher syndrome refers to recessively inherited disorders with associated hearing loss (HL), retinitis pigmentosa (RP), and, sometimes, vestibular dysfunction. Three clinical subtypes, USH1, -2 , and -3 , are defined with respect to the degree and progression of HL and the presence or absence of vestibular areflexia. Usher syndrome type I (USH1) is the most disabling form and is characterized by congenital and profound HL and vestibular dysfunction. RP develops progressively, with night blindness and restriction of the visual field as the first symptoms, as is also true in the other two subtypes. Five causative genes have been identified for USH1 (MIM 276900), myosin VIIA (MYO7A; MIM 276903), harmonin (USH1C, MIM, 605242), cadherin 23 (CDH23, MIM 605516), protocadherin 15 (PCDH15, MIM 605514), and SANS (USH1G, MIM 607696), involved in USH1B (MIM 276900), USH1C (MIM 276904), USH1D (MIM 601067), USH1F (MIM 602083), and USH1G (MIM 606943), respectively. At least two additional genes, lying at loci USH1E (MIM 602097) and USH1H (MIM 612632), remain to be characterized. ${ }^{1,2}$ The prevalence of all combined types of Usher syndrome has been long estimated to be 1 in 25,000 in studies from the United States and Scandinavia, but recent studies estimate an incidence of 1 in $6000 .^{3}$

Identifying pathogenic USH1 mutations remains laborious, as it is impossible to select a gene to be analyzed on the basis of the symptoms and most likely pathogenic variants remain private or rare (see LOVD-USHbases; https://grenada.lumc. nl/LOVD2/Usher_montpellier/USHbases.html/ provided by the Usher Group, Montpellier, France), with the exception of a 
few mutations that are more prevalent in specific populations because of founder effects. A genotyping microarray has been developed by Asper Ophthalmics (Tartu, Estonia), ${ }^{4}$ but the sensitivity is estimated to be $0.5,{ }^{3}$ leaving numerous unsolved diagnostic cases with either both or a single mutation undetected. Our group developed a strategy that includes preliminary haplotype analysis before sequencing of the candidate gene $(s)^{5}$ that has been since upgraded with a systematic multistep analysis involving new technological developments and interpretative tools.

We present, in this study, data obtained after 4 years of USH1 molecular studies using this approach. Seventy-eight USH1 mutations were identified among 61 patients, and $32 \%$ of them are newly described here. Together with our previous work, we found that 92 USH1 patients carried mutations in a USH1 gene. We emphasize several factors that are crucial for a proper diagnostic service.

\section{Patients and Methods}

\section{Patients}

Patients were referred from medical genetic clinics and ophthalmology and ENT services distributed throughout France. In addition four patients were referred from medical genetics clinics in Italy and the United Kingdom.

The parents were available in $70 \%$ of the cases. All patients had audiograms, fundus examination (FE), and/or electroretinograms (ERGs), with the exception of one (patient U379-1). Usher type 1 was diagnosed on the basis of congenital profound sensorineural deafness, vestibular dysfunction, and retinal degeneration. The degree of RP varied among the patients.

This study was approved by the local Ethics Committee and was conducted in accordance with the Declaration of Helsinki. Informed consent for genetic testing was obtained from adult probands or parents, in the case of minors, after explanation of the nature of the study and its possible implications to patients and families.

Patients were mainly Caucasian, but were also North African, Guinean, and Pakistani.

\section{Molecular Analyses}

Haplotyping at the five USH1 loci (USH1B, USH1D, USH1F, USH1C, and USH1G) and sequencing analyses of the five USH1 genes (MYOTA, CDH23, PCDH15, USH1C, and USH1G) have been described. ${ }^{5}$

Several approaches have been used to characterize the large genomic rearrangements: (1) semiquantitative assays were performed by quantitative multiplex PCR of short fluorescent fragments (QMPSF) and semiquantitative nonfluorescent multiplex $\mathrm{PCR}^{6}$ adapted to MYO7A gene rearrangements; (2) multiplex ligation-dependent probe amplification (MLPA) has been designed by MRC-Holland (Amsterdam, The Netherlands) for the PCDH15 gene. This kit (SALSA MLPA kit 292-A1 PCDH15; MRC-Holland) was used according to the manufacturer's recommendations to detect $P C D H 15$ rearrangements; (3) a CGH-microarray chip $(12 \times 135 \mathrm{k})$, laboratory designed and including the Usher genes, was used on a high-resolution microarray platform according to the manufacturer's recommendations (Nimblegen; Roche Diagnostics, Basel, Switzerland) and allowed the identification of the CDH23 exon 20 duplication. The CGH-microarray chip includes 49,144 probes covering all Usher genes (except CLRN1) and their $10,000-\mathrm{bp} 5^{\prime}$ and $3^{\prime}$ regions. The average probe length is 60 bases. The average spacing between starts of the overlapping probes (inner spacing) covering the exons and their 100-bp intronic borders is $10 \mathrm{bp}$, and spacing between the adjacent probes (outer-spacing) covering the introns and the $5^{\prime}$ and $3^{\prime}$ regions is $40 \mathrm{bp}$.

\section{In Silico Studies}

Software used to predict potential splicing alterations has been detailed previously. ${ }^{7,8}$ The multistep analysis for determining the pre- dicted effect of alteration of a variant on protein structure has been described. ${ }^{9}$

The National Center for Biotechnology (NCBI) RefSeq IDs were MYO7A, 4647; CDH23, 64072 (with the initiation codon located in exon 1); PCDH15, 65217; USH1C, 10083; and USH1G, 124590 (available at www.ncbi.nlm.nih.gov/locuslink/refseq/ NCBI, Bethesda MD).

\section{Results}

\section{Haplotype Analyses}

Haplotype analyses were systematically performed to prioritize the gene to be sequenced. If several sibs were available, one or more loci could be excluded by a simple linkage approach. Haplotypes were also useful in simplex cases to look for homozygosity at a locus. ${ }^{5}$

Homozygosity for one locus was revealed in 18 families, and the corresponding gene was sequenced, allowing the identification of the homozygous pathogenic genotype in all cases (12 for $M Y O 7 A, 3$ for $C D H 23,2$ for $P C D H 15$, and 1 for $U S H 1 C$; Table 1). At least one locus could be excluded in an additional six cases (U649, U773, U909, U439, U331, U468, and U322; haplotype analyses not shown). Therefore, similar to our previous data, haplotyping proved its usefulness in $44 \%(25 / 59$ families) of the cases.

\section{Mutation Analysis}

Seventy-eight mutations and likely pathogenic variants are reported in the USH1 genes in Table 2. Twenty-five of them are newly identified. Together with our previous report ${ }^{5}$ a total of $58(48+10)$ different mutations have been identified in $M Y O 7 A, 28(19+9)$ in $C D H 23,16(7+9)$ in $P C D H 15$, and 6 $(3+3)$ in $U S H 1 C$. Mutations are of all types and include premature termination codons (PTCs) due to nucleotide substitutions, small deletions or insertions, missense and translationally silent substitutions (exonic synonymous changes and intronic variations), large genomic rearrangements (that involve at least one-exon), and in-frame deletions. Mutations leading to PTCs or involving large rearrangements are deemed a priori to be deleterious. Any new translationally silent (synonymous) substitution or missense change is considered initially to be an unclassified variant (UV) or a variant of unknown clinical significance and therefore requires special attention to assess its potential pathogenic effect before categorizing it, or not, as likely to be pathogenic (UV3). The multistep analysis, presented in Figure 1, takes into account the clinical and biological context, potential alteration of pre-mRNA splicing, and, when appropriate, the potential effect on the native protein structure and conformation.

\section{Summary of Mutations}

The MYO7A gene shows the highest rate, 36\% (21/58), of nucleotide substitutions leading to missense (Table 2). Indeed, three so-called missense variants were predicted to alter normal splicing of pre-mRNA-p.Ala198Thr, p.Gly1982Arg, and p.Lys400Asn, confirmed in the first two cases by minigene analysis. $^{5,7}$ They are not considered missense changes. $\mathrm{CDH} 23$ contains the highest rate, $30 \%(8 / 28)$, of splicing alterations (Table 3). In addition, half the $U S H 1 C$ mutations (3/6) result in aberrant splicing. Some remove the canonical AG/GT sites, but some also lie in the introns, outside the invariant sites, or correspond to exonic nucleotide substitutions. Ex vivo assays and transcript analyses from nasal cells for $C D H 23$ c.6050$9 \mathrm{G}>\mathrm{A}$ and ex vivo assays for $U S H 1 C$ c. $1210+6 \mathrm{~T}>\mathrm{C}$ provided evidence for deleterious outcomes with the creation of PTC, either by use of a created acceptor site $e^{8,25}$ in the first case or by activation of an upstream exonic cryptic splice site in the 
Table 1. Pathogenic Genotypes of the Families in the MYO7A, CDH23, PCDH15, USH1C Genes

\begin{tabular}{|c|c|c|}
\hline Gene & Family & Genotype \\
\hline \multirow[t]{42}{*}{$M Y O 7 A$} & U105 & c. $[3719 \mathrm{G}>\mathrm{A}]+[3979 \mathrm{G}>\mathrm{A}]$ \\
\hline & U107 & c. $[5632 \mathrm{delC}]+[2513 \mathrm{G}>\mathrm{A}]$ \\
\hline & U139* & c. $\left[2874 \_2878 d e l C C A G G\right]+\left[2874 \_2878 d e l C C A G G\right]$ \\
\hline & U194* & c. $[5573 \mathrm{~T}>\mathrm{C}]+\left[1157 \_1158 \mathrm{delTG}\right]$ \\
\hline & U299† & c. $\left[5886 \_5889\right.$ delCTTT $]+[5856+1 \mathrm{G}>\mathrm{A}]$ \\
\hline & U379-3t & c. $[5392 \mathrm{C}>\mathrm{T}]+[493 \mathrm{~A}>\mathrm{G}]$ \\
\hline & U379-2 & c. $[493 \mathrm{~A}>\mathrm{G}]+[3502 \mathrm{C}>\mathrm{T}]$ \\
\hline & U379-1 & c. $[5392 \mathrm{C}>\mathrm{T}]+[3476 \mathrm{G}>\mathrm{T}]$ \\
\hline & $\mathrm{U} 407 \dagger$ & c. $[1303 \mathrm{delC}(+) 2797 \mathrm{delC}]$ \\
\hline & U419* & c. $[640 G>A]+[5573 \mathrm{~T}>\mathrm{C}]$ \\
\hline & U437 & c. $[1555-8 \mathrm{C}>\mathrm{G}]+[3719 \mathrm{G}>\mathrm{A}]$ \\
\hline & $\mathrm{U} 445$ & c. $[2005 \mathrm{C}>\mathrm{T}]+[2005 \mathrm{C}>\mathrm{T}]$ \\
\hline & U492 & c. $[2283-1 G>T]+[2283-1 G>T]$ \\
\hline & U495 & c. [6354+628_*737del] + [6_9dup] \\
\hline & U506† & c. $\left[4648 \_4852+668 \mathrm{del}\right]+\left[4648 \_4852+668 \mathrm{del}\right]$ \\
\hline & U520 & c. $\left[2283-1 \mathrm{G}>\mathrm{T}(+) 5886 \_5888 \mathrm{delCTT}\right]$ \\
\hline & U570† & c. $[6025 \mathrm{delG}]+[5004 \mathrm{C}>\mathrm{G}]$ \\
\hline & U590 & c. $\left[5886 \_5888 \mathrm{delCTT}\right]+\left[5886 \_5888 \mathrm{delCTT}\right]$ \\
\hline & U597 & c. $[5434 \mathrm{G}>\mathrm{A}]+[5434 \mathrm{G}>\mathrm{A}]$ \\
\hline & U599 & c. $[3702 \mathrm{delC}(+) 5617 \mathrm{C}>\mathrm{T}]$ \\
\hline & U649 & c. $[397 \mathrm{C}>\mathrm{T}]+[5944 \mathrm{G}>\mathrm{A}]$ \\
\hline & $\mathrm{U} 662$ & c. $[2513 \mathrm{G}>\mathrm{A}]+[2513 \mathrm{G}>\mathrm{A}]$ \\
\hline & U653A & c.[999T>G(+)NM_004055.4:c.165+3559_c.5168+213del] \\
\hline & $\mathrm{U} 653 \mathrm{~B}$ & c. $[1954 \mathrm{delT}(+) 5101 \mathrm{C}>\mathrm{T}]$ \\
\hline & U700 & c. $[3719 \mathrm{G}>\mathrm{A}]+[5623 \mathrm{C}>\mathrm{T}]$ \\
\hline & U707 & c. $[2461 \mathrm{C}>\mathrm{T}(+) 3764 \mathrm{delA}]$ \\
\hline & U733 & c. $[2283-1 G>T]+[2283-1 G>T]$ \\
\hline & $\mathrm{U} 742$ & c. $[6025 \mathrm{delG}]+[6025 \mathrm{delG}]$ \\
\hline & $\mathrm{U} 750$ & c. $[1200 \mathrm{G}>\mathrm{T}(+) 6025 \mathrm{delG}]$ \\
\hline & U766 & c. $[1555-8 \mathrm{C}>\mathrm{G}(+) 5392 \mathrm{C}>\mathrm{T}]$ \\
\hline & $\mathrm{U} 773$ & c. $[6062 A>G]+[722 G>A]$ \\
\hline & $\mathrm{U} 779$ & c. $[3719 \mathrm{G}>\mathrm{A}(+) 5617 \mathrm{C}>\mathrm{T}]$ \\
\hline & U803 & c. $\left[4117 \mathrm{C}>\mathrm{T}(+) 5750 \_* 2614 \mathrm{del}\right]$ \\
\hline & U805 & c. $[3719 \mathrm{G}>\mathrm{A}(+) 6025 \mathrm{delG}]$ \\
\hline & U811 & c. [3594C>A;4036_4038delTTC]+[494C>T] \\
\hline & $\mathrm{U} 812$ & c. $[3508 G>A]+[6025 \mathrm{delG}]$ \\
\hline & U822 & c. $[3508 \mathrm{G}>\mathrm{A}]+[3508 \mathrm{G}>\mathrm{A}]$ \\
\hline & U842 & c. $[487 \mathrm{G}>\mathrm{A}]+[3979 \mathrm{G}>\mathrm{A}]$ \\
\hline & U866 & c. $[2283-1 G>T]+[2283-1 G>T]$ \\
\hline & U887 & c. $[2283-1 G>T]+[2283-1 G>T]$ \\
\hline & U898 & c. $[3594 \mathrm{C}>\mathrm{A}(+) 3719 \mathrm{G}>\mathrm{A}]$ \\
\hline & U909 & c. $[700 \mathrm{C}>\mathrm{T}]+[6557 \mathrm{~T}>\mathrm{C}]$ \\
\hline \multirow{12}{*}{$\mathrm{CDH} 23$} & U93 & c. $[5985 \mathrm{C}>\mathrm{A}]+[6050-9 \mathrm{G}>\mathrm{A}]$ \\
\hline & U189† & c.[1987-2A >C] + [6146_6153del; NM_206933.2:c.2299delG] \\
\hline & $\mathrm{U} 447 \dagger$ & c. $\left[3713 \_3714\right.$ delCT $]+[5821-2 \mathrm{~A}>\mathrm{G}]$ \\
\hline & U439 & c. $[4069 \mathrm{C}>\mathrm{T}]+\left[2177-104 \_2290-313 \mathrm{dup}\{\mathrm{insA}\}\right]$ \\
\hline & $\mathrm{U} 453 \dagger$ & c. $[427 \mathrm{G}>\mathrm{C}]+[272 \mathrm{delA}]$ \\
\hline & U499† & c. $[2587+1 \mathrm{G}>\mathrm{A}]+[2587+1 \mathrm{G}>\mathrm{A}]$ \\
\hline & $\mathrm{U} 507^{*}$ & c. $[3367 \mathrm{C}>\mathrm{T}]+[3580-1 \mathrm{G}>\mathrm{T}]$ \\
\hline & U514† & c. $[7872 \mathrm{G}>\mathrm{A}]+[7026 \mathrm{delG}]$ \\
\hline & $\mathrm{U} 562$ & c. $\left[790 \mathrm{G}>\mathrm{T}(+) 8054 \_8055 \mathrm{delCG}\right]$ \\
\hline & $\mathrm{U} 752$ & c. $[9167 \mathrm{delT}]+[9167 \mathrm{delT}]$ \\
\hline & U826 & c. $[6050-9 G>A]+[6050-9 G>A]$ \\
\hline & U884 & c. $\left[3367 \mathrm{C}>\mathrm{T}(+) 4759 \_4768 \mathrm{del}\right]$ \\
\hline \multirow[t]{5}{*}{ PCDH15 } & $\mathrm{U} 331$ & {$[?]+[c .2092-? 3501+? \mathrm{del}]$} \\
\hline & $\mathrm{U} 468$ & c. $[407 \mathrm{~T}>\mathrm{C}]+[3807-?-4367+? \mathrm{del}]$ \\
\hline & $\mathrm{U} 322$ & c. $\left[7 \mathrm{C}>\mathrm{T}(+) \mathrm{c} .92-? \_157+? \mathrm{del}\right]$ \\
\hline & U834 & c. $[2971 \mathrm{C}>\mathrm{T}(+) 2971 \mathrm{C}>\mathrm{T}]$ \\
\hline & U877 & c. $[3373+1 \mathrm{G}>\mathrm{A}]+[3373+1 \mathrm{G}>\mathrm{A}]$ \\
\hline \multirow[t]{2}{*}{ USH1C } & U360 & c. $[1084 \mathrm{C}>\mathrm{T}(+) 1210+6 \mathrm{~T}>\mathrm{G}]$ \\
\hline & U819 & c. $[216 \mathrm{G}>\mathrm{A}]+[216 \mathrm{G}>\mathrm{A}]$ \\
\hline
\end{tabular}

U379 family consists of the two parents (U379-1 and U379-2) and 1 child (U379-3) with different phenotypes and was therefore considered for calculations as a single family but 3 patients. U653A and U653B were referred as a couple and were therefore considered as two families for calculations.

* Families already reported in Blanchet et al. ${ }^{10}$

† Mutations carried by these patients were included in Baux et al. ${ }^{11}$ Genotypes are presented according to HGVS nomenclature. Therefore, when segregation analysis could not be performed the uncertain status is indicated with $(+)$. 


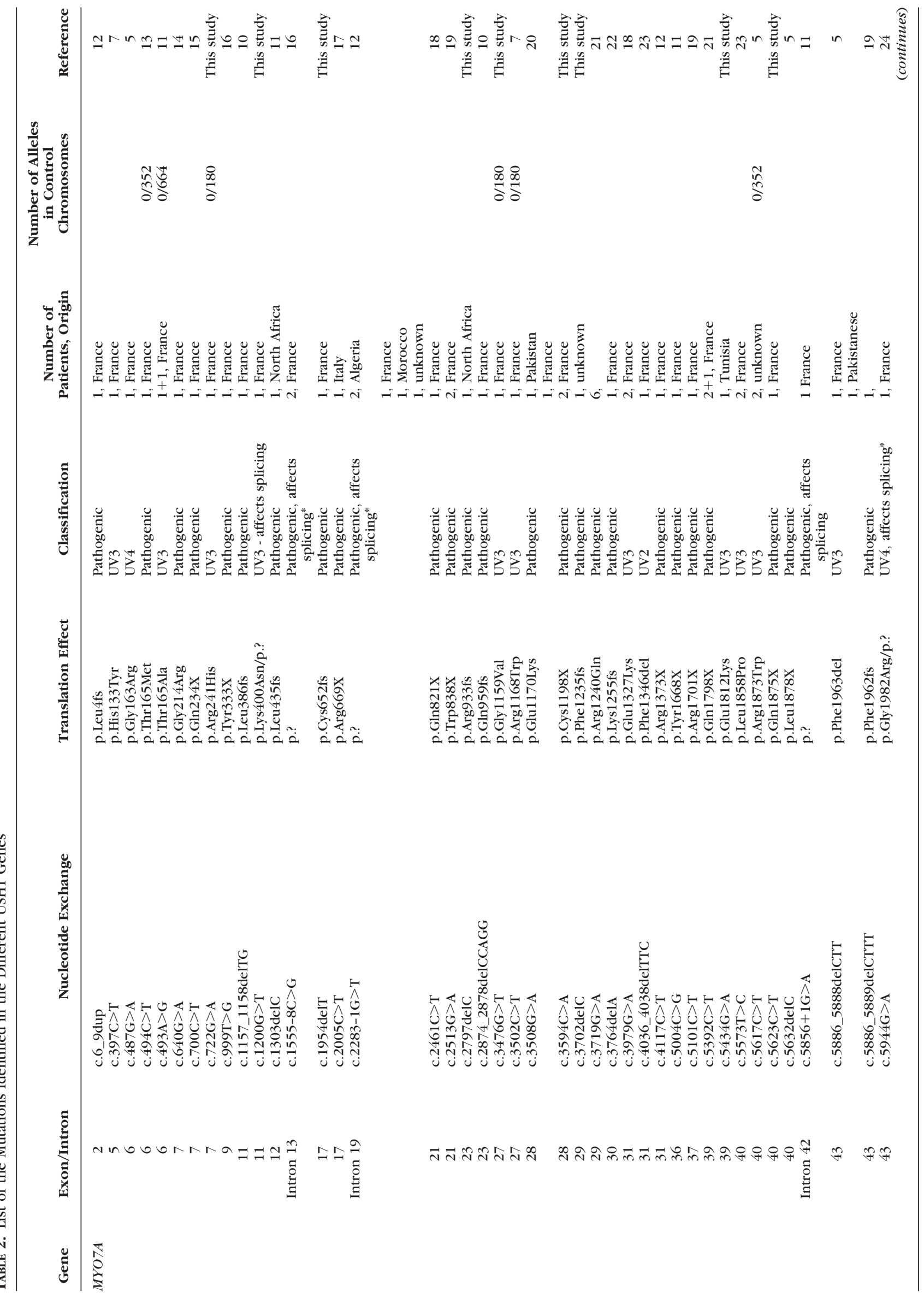




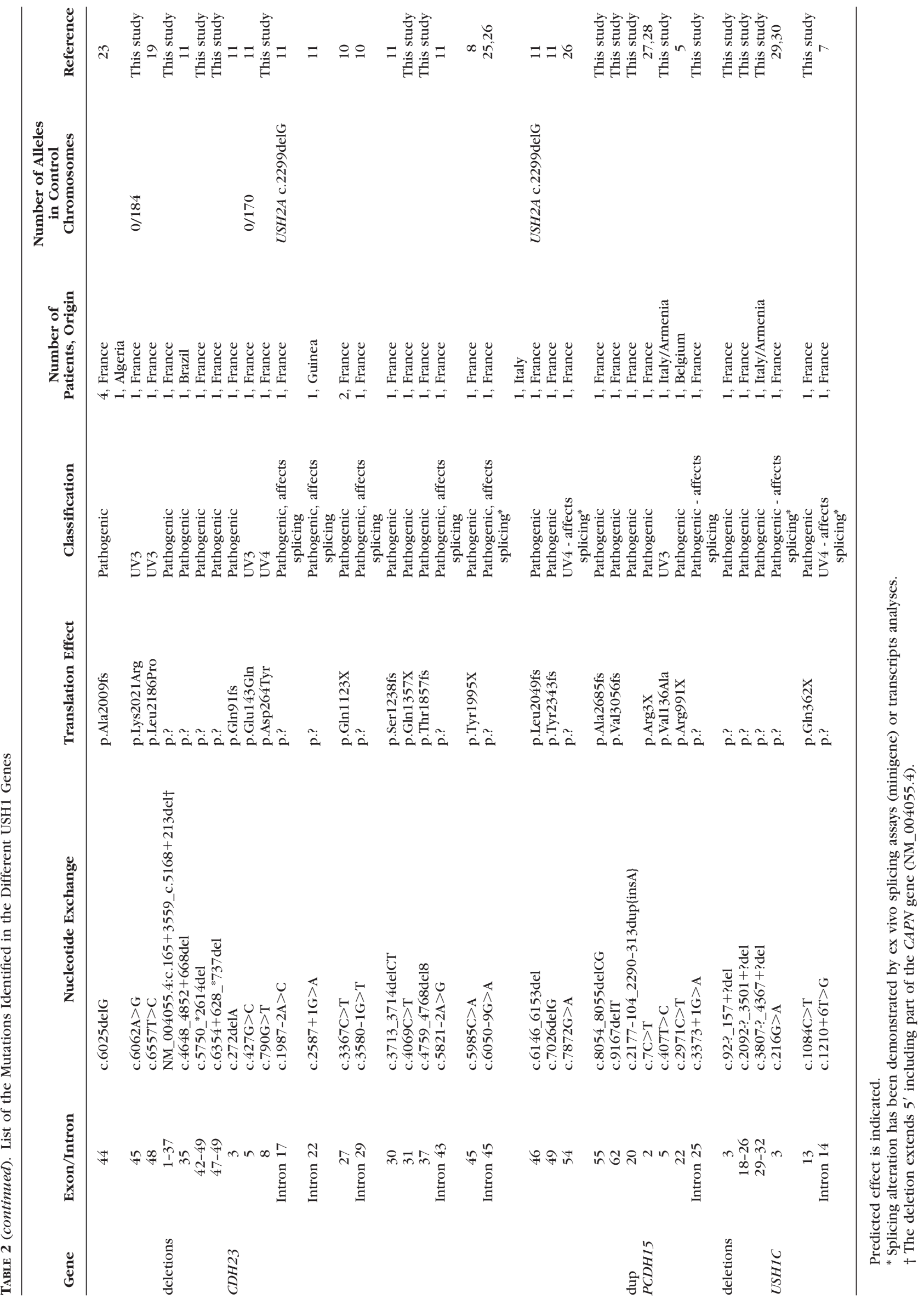




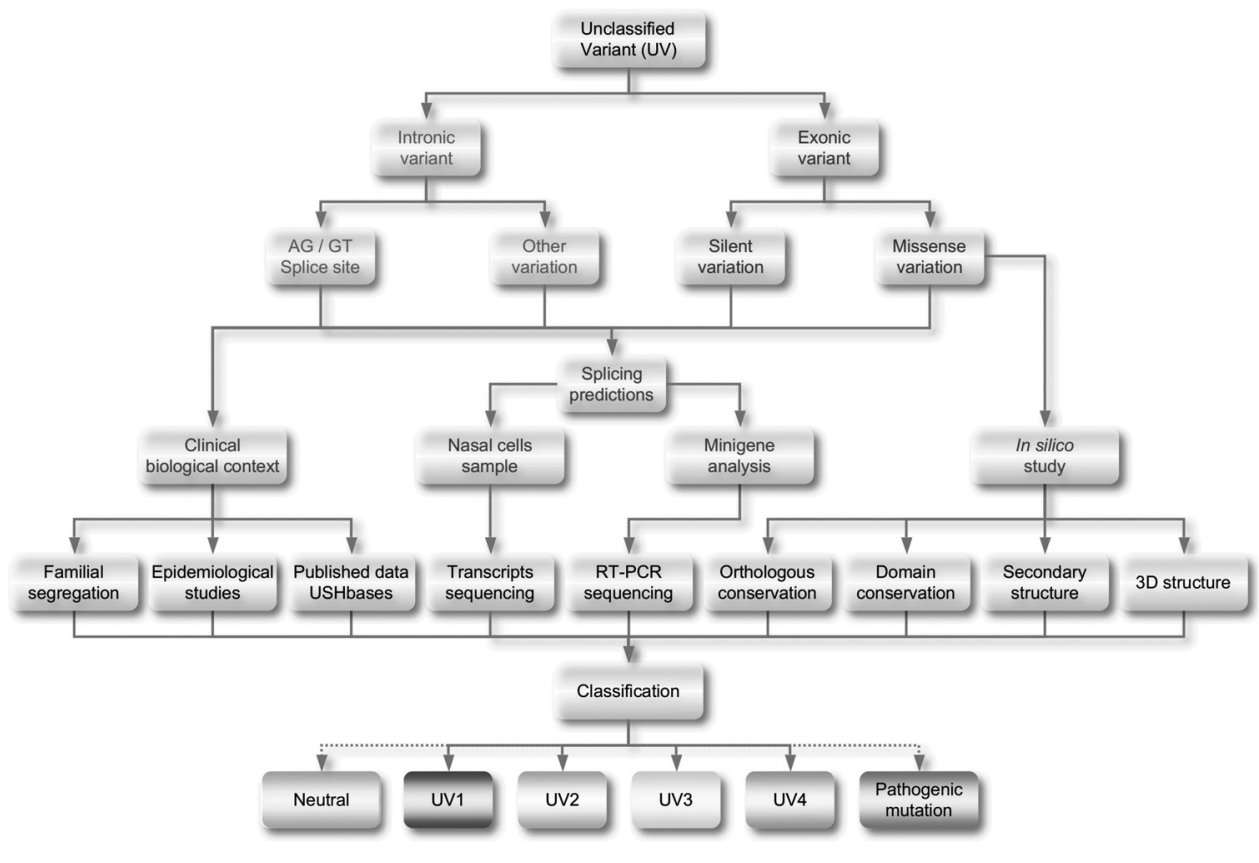

Figure 1. Multistep analysis applied in the diagnostic procedure to classify UVs. Depending on the nature of the UV (exonic, intronic, silent, or missense), different analyses are performed and information collected before final classification. latter. ${ }^{7}$ The $U S H 1 C$ c. $216 \mathrm{G}>\mathrm{A}$ variant was previously shown to be an in-frame ${ }^{29}$ or an out-of-frame ${ }^{31}$ splice site mutation. Of note, nasal epithelial cell analyses of U819 revealed the simultaneous presence of both effects. Finally, our data confirm that $P C D H 15$ is particularly prone to large rearrangements, as they represent $37 \%(6 / 16)$ of the mutations.

The presence of large genomic rearrangements is considered in all cases when either no mutation in any of the five USH1 has been detected, or a single mutation at the UV3 level has been identified in one of the USH1 genes.

\section{Assessment of the Pathogenic Effect of the Missense Alterations}

Several lines of evidence (see Fig. 1) are used to classify the UVs. ${ }^{5,9}$ Table 2 includes only variants that have been classified as likely to be pathogenic (i.e., corresponding to UV3 and UV4, according to the CMGS guidelines (url: http://www.cmgs.org/ provided by the Clinical Molecular Genetics Society, a subsidiary body of the British Society for Human Genetics), with the exception of the MYO7A F1346del variant classified as UV2, mainly because it has been characterized in a complex allele, together with the deleterious p.Cys1198X mutation (patient U811; Table 1).

TABLE 3. Distribution of the Different Types of Alterations (Pathogenic Mutations UV4 and UV3) among the USH1 Genes

\begin{tabular}{lrccc}
\hline & MYO7A & CDH23 & PCDH15 & USH1C \\
\hline Missense & 21 & 3 & 1 & 1 \\
PTC* & 26 & 16 & 8 & 2 \\
Splicingt & 5 & 8 & 1 & 3 \\
Small deletion in-frame & 1 & & & \\
Large rearrangements & 4 & 1 & 6 & 0 \\
Total & 57 & 28 & 16 & 6 \\
\hline
\end{tabular}

The calculation includes the data presented in Roux et al. ${ }^{5}$

* Premature stop codons (PTCs) due to nucleotide substitution and small deletions and insertions.

† Splicing defects predicted or demonstrated by ex vivo splicing assays (minigenes) and/or transcripts studies from nasal epithelial cells.
Analyses at the protein level are performed according to a multistep process (Fig. 1). The steps have been combined as a new tool dedicated to missense in Usher genes, USMA (Usher Syndrome Missense Analysis, currently in beta version for public use, available at https://194.167.35.160/cgi-bin/USMA/USMA. fcgi).

\section{Discussion}

\section{Diagnostic Approach}

Together with published studies from our laboratory, ${ }^{5-8,10,11}$ we provide evidence of a powerful service for patients. A total of 92 patients have received diagnoses, with no ambiguous USH1 genotypes.

These 4 years of service confirm that the chosen approach (i.e., preliminary haplotyping before gene sequencing) is efficient, as it helped in $44 \%$ of the families to prioritize the gene to be sequenced. For example, this strategy proved to be efficient for U819. As homozygosity was found at the USH1C locus, only USH1C was sequenced to identify the homozygous causative mutation. For uninformative families, the genes were sequenced following their relative involvement. This effort was laborious and time consuming for $\mathrm{U} 360$, as $M Y O 7 A, C D H 23$, and $P C D H 15$ had to be sequenced before identifying the pathogenic USH1C genotype. The finding of homozygosity was frequently useful, even in cases without any indication of consanguinity. Interestingly, this empiric finding has recently been used to identify a new retinal-renal ciliopathy gene. ${ }^{32}$

The spectrum of mutations is notable for the high proportion of private mutations. Twenty-five are reported here for the first time (i.e., 32\%). To these, an additional 18 private variants can be added that were published separately by our group in the course of the creation of USHbases, ex vivo assays and nasal cell transcript studies.,8,11 Only a few founder effects have been observed-for example the $\mathrm{CDH} 23$ p.Arg1502X mutation in Swedes, ${ }^{26}$ PCDH15 p.Arg245X in Ashkenazi Jews, and USH1C c. $216 \mathrm{G}>\mathrm{A}$ in Acadians ${ }^{29,30}$ and Quebecois. ${ }^{33}$ As a consequence the Asper chip based on known mutations would have been inefficient and would have solved only $52 \%$ of the cases. 
New technologies allowing large scale sequencing of exomes $^{32,34}$ will become available to diagnostic services as costs reduce further, probably in the comparatively near future. Preliminary capture of the 5 USH1 genes, including intronic sequences, should be fairly straightforward. As well as simple technical problems (difficulty of finding genome rearrangements, or small del/ins) the results will elicit issues of interpretation. On the positive side they will produce evidence for some complex genotypes that may be related to variable phenotypes thus providing clues to overlapping phenotypes (socalled atypical Usher). They will also reveal probably rare digenic cases, as has been recently shown in USH2 with the GPR98 and PDZD7 genes. ${ }^{35}$ They will also generate high amounts of data that will complicate the interpretation of the results and the message to the families.

It is also clearly demonstrated with the data presented here and previously ${ }^{5}$ that, an efficient diagnostic service must include approaches other than sequencing, such as MLPA, QMPSF, or array-CGH, this is particularly crucial for $P C D H 15$, but also for MYOTA.

The service must also have access to tools for proper interpretation of the different variants identified in the course of the analysis. These tools require expertise in bioinformatics. In diagnostics, consideration of the splicing effect of translationally silent or missense alterations is crucial for correct classification of gene alterations..$^{7,36,37}$ Numerous splicing prediction software programs are available and have been reviewed by Spurdle et al. ${ }^{38}$ Although useful, the outcome of the splicing default is still difficult to predict, and ex vivo assays and splicing studies from RNA are therefore preferable.

We compile the maximum information available for a given variant (orthologue analysis, alignment of protein domains, secondary structure, and 3D predictions), which we consider to be more informative than a predictor program, as many are heavily based on alignments. ${ }^{39}$ We designed software to fulfill our needs, called USMA (Baux D, unpublished, 2010, see the Results section). Another benefit of customized assessment is that sometimes it is possible to envisage the molecular mechanism responsible for protein function and pathogenicity.

\section{Complex Genotypes and Phenotypes}

Family U379 had undergone several investigations, as the father (U379-1) was diagnosed with congenital profound deafness and the mother (U379-2) with mild-to-severe deafness and late RP, fortuitously diagnosed at the age of 24 . However, the daughter (U379-3) had typical USHI signs (profound deafness, vestibular areflexia, and RP onset at the age of 12). In view of the variety of symptoms, several types of transmission had been hypothesized, including dominant and mitochondrial inheritance. When the family was referred to our laboratory, we considered the known involvement of some Usher genes in NSHL as well as variable clinical manifestations and decided to screen the $M Y O 7 A$ gene. The genotypes proved to be quite challenging to interpret. The p.Thr165Ala mutation was identified for the first time in this patient. Residue Thr165 has been implicated as a recessive mutation (p.Thr165Met). It is part of the ATP binding cluster GESGAGKT[EV] of the myosin VIIA protein (Fig. 2) ${ }^{40}$ which is particularly conserved among myosins. ${ }^{41}$ All variants identified at positions 163 to 165 (residues GKT) are considered to act as recessive alterations (see below; LOVD-USHbases). It was therefore logical to regard p.Thr165Ala as a likely pathogenic recessive alteration.

The clinical variability in this family is not clearly understood. The father (U379-1) suffers from NSHL, although no additional ophthalmic exploration could be performed to exclude the RP phenotype, but he did not complain of night blindness or restricted visual fields at the age of 56. The

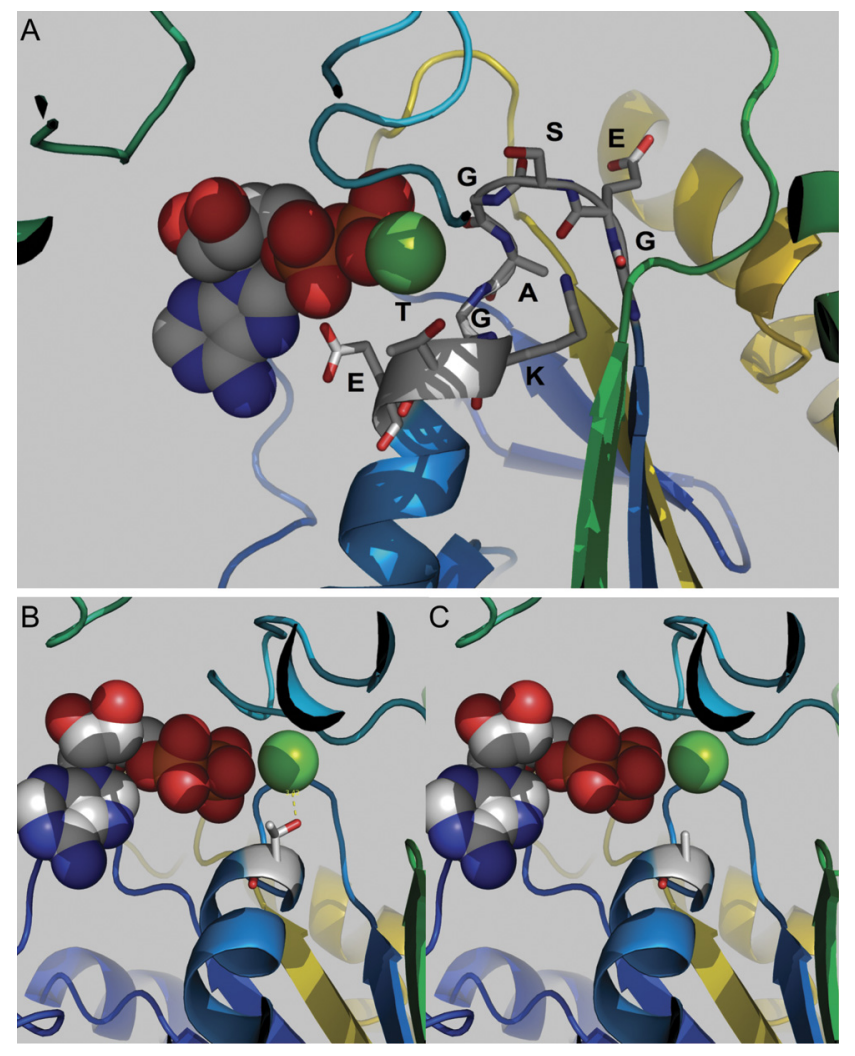

Figure 2. 3D modeling of myosin VIIa Sticks corresponds to aminoacids within the partial protein (model build from the PDB (Protein Data Bank template 1G8X [www.pdb.org], 36\% identity between target and template, using the method described in Ref. 9). Green sphere: an $\mathrm{Mg}^{2+}$ atom, and the other spheres are the atoms from ADP. (A) Predicted representation of the GESGAGKT[EV] motif, highly conserved in myosins, corresponding to residues 158 to 166 in myosin VIIa. (B) Wild-type Thr165 is likely to be in direct interaction with $\mathrm{Mg}^{2+}$. (C) Replacement of Thr with Ala would disrupt this interaction.

common cause of NSHL by DFNB1 mutations was excluded, as well as the presence of the mitochondrial A1555G change. He carries the MYO7A genotype p.[Gly1159Val]+[Gln1798X]. The mother, presenting with atypical USH1, carries p.Thr165Ala in trans to c.3502C $>$ T (p.Arg1168Trp). Interestingly, the neighboring substitution c.3503G $>C$ (predicted p.Arg 1168 Pro $)^{12}$ has been shown to be acting at splicing level, as it induces the skipping of exon 27 , whereas c.3502C $>\mathrm{T}$ does not. ${ }^{7}$ Therefore, any likely pathogenic effect is due to the Arg to Trp change. Using our standard techniques, we have no explanation for why both parents display a milder phenotype, and this is consistent with the clinical variability of $M Y O 7 A$ mutations previously observed. ${ }^{24,42}$

\section{Patient U189}

This young patient was referred with diagnosed clinical USH1, presenting with particularly early ophthalmic symptoms. Nystagmus at the age of 3 months and abnormal ERG led to the diagnosis of RP at the age of 1 year. The child also had some balance problems, but walked at 18 months. Profound deafness was diagnosed at 3 years. Molecular analysis found two CDH23 deleterious mutations. During USH2A c.2299delG random screening in USH patients and controls for epidemiologic studies to establish the carrier frequency, ${ }^{9}$ a c.2299delG mutation was revealed in the patient that was inherited from the mother. Perhaps $U S H 2 A$ acts as a modifier of the RP phenotype in this patient on a $\mathrm{CDH} 23$ background, similarly to what has 
been recently demonstrated for $P D Z D 7$ acting as a modifier on a $U S H 2 A$ background. ${ }^{35}$ The mother was double heterozygous for CDH23 c.6146_6153del and USH2A c.2299delG. Unfortunately, additional clinical explorations could not be performed in the mother, but she had no apparent Usher-linked symptoms.

The practical use of our diagnostic service was shown for couple U653A and U653B. Both patients, affected with typical USH1, wanted to know their risk of having an affected child. Both of them carried two deleterious mutations in the $M Y O 7 A$ gene, leading to unambiguous genetic counseling.

\section{Patient U331}

Patient U331 (typical USH1) was referred with two nonaffected siblings. Linkage analysis at the different USH loci excluded $M Y O 7 A$. All the other USH1 genes were sequenced, and a single deleterious mutation in PCDH15 (deletion of exons 18-26) was found. We also excluded mutations in CLRN1, involved in USH3, known to overlap with USH1 phenotype. Recently additional alternative spliced exons have been identified, ${ }^{43}$ and their analysis revealed a new missense alteration (NM_001142769.1:c.4853A $>$ C, NP_001136241.1: p.Glu1618Ala, in exon 38 of isoform CD2.1) allelic to the E18-26 deletion. It is likely that a second $P C D H 15$ alteration remains undetectable, lying in unscreened regions (i.e., located deep in the introns or in the regulatory regions or because of allele dropout [due to an SNP located on one of the used primers that is not described in SNP databases]). Digenism cannot be excluded; $P C D H 15 / C D H 23$ digenic inheritance has been documented, ${ }^{44}$ but that was before the awareness that large rearrangements in $\mathrm{PCDH} 15$ were involved in a non-negligible proportion. ${ }^{6,45} \mathrm{CDH} 23$ has been sequenced in the course of the study, and no pathogenic mutation or UV could be identified. Unfortunately, this family still has a partial genotype, and additional investigations are necessary.

\section{USHbases}

All the USH1 alterations presented in this study were incorporated into the USHbases, as well as all likely nonpathogenic and neutral variants found among the patients. These databases, previously using the UMD software, ${ }^{11}$ have recently been updated using the LOVD open-source system. They are now available at https://grenada.lumc.nl/LOVD2/Usher_montpellier/USHbases.html, and databases for WHRN and GPR98 have been added.

Thanks to this study, the database number of pathogenic variants has been raised from 189 to 201 for $M Y O 7 A$ and from 352 to 377 for the five USH1 genes.

Use of databases is becoming crucial in diagnosis for pooling data and sorting genotypes and for interpretation. By integrating all the records into databases, this study shows once more the powerful source of data that diagnostic services can offer, not only to the patients and their families but also to the scientific community and other diagnostic laboratories.

\section{Contribution of the USH Genes/Overall Mutation Detection Rate}

Among the 31 USH1 families previously published and the 59 reported in this study, MYO7A was implicated in $63.3 \%$, CDH23 in $20 \%, P C D H 15$ in $12.2 \%$, and $U S H 1 C$ in $4.5 \%$. $M Y O 7 A$ was predominant, but one in three cases involved one cadherin gene. USH1C was only rarely involved in our cohort, whereas we have never identified any pathogenic variants in USH1G.
Overall, the mutation detection rate was greater than $90 \%$. Of the few patients in whom we were unable to establish a molecular cause, at least some had an atypical phenotype.

\section{Acknowledgments}

The authors thank the families who participated in this study and Clarisse Baumann, Maria Bitner-Glindzicz, Patricia Blanchet, Pierangela Castorina, Christine Francannet, Anne-Marie Frances, Alice Goldenberg, Georges Haddad, Koenraad Devriendt, Sandrine Marlin, Dominique Martin-Coignard, Philippe Parent, Annick Rossi, Sabine Sigaudy, Renaud Touraine, Annick Toutain, and Jacqueline Vigneron for referring patients and Philippe Khau Van Kien and Christophe Béroud for initial design of the CGH array.

\section{References}

1. Petit C. Usher syndrome: from genetics to pathogenesis. Annu Rev Genomics Hum Genet. 2001;2:271-297.

2. Saihan Z, Webster AR, Luxon L, Bitner-Glindzicz M. Update on Usher syndrome. Curr Opin Neurol. 2009;22:19-27.

3. Kimberling WJ, Hildebrand MS, Shearer AE, et al. Frequency of Usher syndrome in two pediatric populations: implications for genetic screening of deaf and hard of hearing children. Genet Med. 2010;12:512-516.

4. Cremers FP, Kimberling WJ, Kulm M, et al. Development of a genotyping microarray for Usher syndrome. J Med Genet. 2007; 44:153-160.

5. Roux AF, Faugère V, Le Guédard S, et al. Survey of the frequency of USH1 gene mutations in a cohort of Usher patients shows the importance of cadherin 23 and protocadherin 15 genes and establishes a detection rate of above 90\%. J Med Genet. 2006;43:763768.

6. Le Guédard S, Faugère V, Malcolm S, Claustres M, Roux AF. Large genomic rearrangements within the PCDH15 gene are a significant cause of USH1F syndrome. Mol Vis. 2007;13:102-107.

7. Le Guédard-Mereuze S, Vaché C, Baux D, et al. Ex vivo splicing assays of mutations at non-canonical positions of splice sites in USHER genes. Hum Mutat. 2010;31:347-355.

8. Vaché C, Besnard T, Blanchet $\mathrm{C}$, et al. Nasal epithelial cells are a reliable source to study splicing variants in Usher syndrome. Hum Mutat. 2010;31:734-741.

9. Baux D, Larrieu L, Blanchet C, et al. Molecular and in silico analyses of the full-length isoform of usherin identify new pathogenic alleles in Usher type II patients. Hum Mutat. 2007;28:781-789.

10. Blanchet C, Roux AF, Hamel C, et al. Usher type I syndrome in children: genotype/phenotype correlation and cochlear implant benefits (in French). Rev Laryngol Otol Rbinol (Bord). 2007;128: 137-143.

11. Baux D, Faugere V, Larrieu L, et al. UMD-USHbases: a comprehensive set of databases to record and analyse pathogenic mutations and unclassified variants in seven Usher syndrome causing genes. Hum Mutat. 2008;29:E76-E87.

12. Jaijo T, Aller E, Oltra S, et al. Mutation profile of the MYO7A gene in Spanish patients with Usher syndrome type I. Hum Mutat. 2006;27:290-291.

13. Ouyang XM, Yan D, Du LL, et al. Characterization of Usher syndrome type I gene mutations in an Usher syndrome patient population. Hum Genet. 2005;116:292-299.

14. Adato A, Weil D, Kalinski H, et al. Mutation profile of all 49 exons of the human myosin VIIA gene, and haplotype analysis, in Usher 1B families from diverse origins. Am J Hum Genet. 1997;61:813821.

15. Levy G, Levi-Acobas F, Blanchard S, et al. Myosin VIIA gene: heterogeneity of the mutations responsible for Usher syndrome type IB. Hum Mol Genet. 1997;6:111-116.

16. Weston MD, Kelley PM, Overbeck LD, et al. Myosin VIIA mutation screening in 189 Usher syndrome type 1 patients. Am J Hum Genet. 1996;59:1074-1083.

17. Weston MD, Carney CA, Rivedal SA, Kimberling WJ. Spectrum of MyosinVIIA mutations causing Usher Syndrome type 1b. Assoc Res Otolaryngol. 1998:Abstract 181. 
18. Najera C, Beneyto M, Blanca J, et al. Mutations in myosin VIIA (MYO7A) and usherin (USH2A) in Spanish patients with Usher syndrome types I and II, respectively. Hum Mutat. 2002;20:76-77.

19. Gerber S, Bonneau D, Gilbert B, et al. USH1A: Chronicle of a slow death. Am J Hum Genet. 2006;78:357-359.

20. Cuevas JM, Espinos C, Millan JM, et al. Identification of three novel mutations in the MYO7A gene. Hum Mutat. 1999;14:181.

21. Janecke AR, Meins M, Sadeghi M, et al. Twelve novel myosin VIIA mutations in 34 patients with Usher syndrome type I: confirmation of genetic heterogeneity. Hum Mutat. 1999;13:133-140.

22. Jaijo T, Aller E, Beneyto M, et al. MYO7A mutation screening in Usher syndrome type I patients from diverse origins. I Med Genet. 2007; 44:e71.

23. Bharadwaj AK, Kasztejna JP, Huq S, Berson EL, Dryja TP. Evaluation of the myosin VIIA gene and visual function in patients with Usher syndrome type I. Exp Eye Res. 2000;71:173-181.

24. Riazuddin S, Nazli S, Ahmed ZM, et al. Mutation spectrum of MYO7A and evaluation of a novel nonsyndromic deafness DFNB2 allele with residual function. Hum Mutat. 2008;29:502-511.

25. von Brederlow B, Bolz H, Janecke A, et al. Identification and in vitro expression of novel $\mathrm{CDH} 23$ mutations of patients with Usher syndrome type 1D. Hum Mutat. 2002;19:268-273.

26. Astuto LM, Bork JM, Weston MD, et al. CDH23 mutation and phenotype heterogeneity: a profile of 107 diverse families with Usher syndrome and nonsyndromic deafness. Am J Hum Genet. 2002;71:262-275.

27. Alagramam KN, Yuan H, Kuehn MH, et al. Mutations in the novel protocadherin PCDH15 cause Usher syndrome type 1F. Hum Mol Genet. 2001;10:1709-1718.

28. Ahmed ZM, Riazuddin S, Bernstein SL, et al. Mutations of the protocadherin gene PCDH15 cause Usher syndrome type 1F. Am J Hum Genet. 2001;69:25-34.

29. Bitner-Glindzicz M, Lindley KJ, Rutland P, et al. A recessive contiguous gene deletion causing infantile hyperinsulinism, enteropathy and deafness identifies the usher type 1C gene. Nat Genet. 2000;26:56-60

30. Verpy E, Leibovici M, Zwaenepoel I, et al. A defect in harmonin, a PDZ domain-containing protein expressed in the inner ear sensory hair cells, underlies Usher syndrome type 1C. Nat Genet. 2000;26: 51-55.

31. Lentz J, Savas S, Ng SS, Athas G, Deininger P, Keats B. The USH1C $216 \mathrm{G} \rightarrow \mathrm{A}$ splice-site mutation results in a 35-base-pair deletion. Hum Genet. 2005;116:225-227.
32. Otto EA, Hurd TW, Airik R, et al. Candidate exome capture identifies mutation of SDCCAG8 as the cause of a retinal-renal ciliopathy. Nat Genet. 2010;42:840-850.

33. Ebermann I, Lopez I, Bitner-Glindzicz M, Brown C, Karel Koenekoop R, Jorn Bolz H. Deafblindness in French Canadians from Quebec: a predominant founder mutation in the USH1C gene provides the first genetic link with the Acadian population. Genome Biol. 2007;8:R47.

34. Ng SB, Turner EH, Robertson PD, et al. Targeted capture and massively parallel sequencing of 12 human exomes. Nature. 2009; 461:272-276.

35. Ebermann I, Phillips JB, Liebau MC, et al. PDZD7 is a modifier of retinal disease and a contributor to digenic Usher syndrome.J Clin Invest. 2010;120:1812-1823.

36. Hartmann L, Theiss S, Niederacher D, Schaal H. Diagnostics of pathogenic splicing mutations: does bioinformatics cover all bases? Front Biosci. 2008;13:3252-3272.

37. Raponi M, Baralle D. Alternative splicing: good and bad effects of translationally silent substitutions. FEBS J. 2010;277:836-840.

38. Spurdle AB, Couch FJ, Hogervorst FB, Radice P, Sinilnikova OM Prediction and assessment of splicing alterations: implications for clinical testing. Hum Mutat. 2008;29:1304-1313.

39. Thusberg J, Vihinen M. Pathogenic or not? and if so, then how?studying the effects of missense mutations using bioinformatics methods. Hum Mutat. 2009;30:703-714.

40. Weil D, Levy G, Sahly I, et al. Human myosin VIIA responsible for the Usher 1B syndrome: a predicted membrane-associated motor protein expressed in developing sensory epithelia. Proc Natl Acad Sci U S A. 1996;93:3232-3237.

41. Cope MJ, Whisstock J, Rayment I, Kendrick-Jones J. Conservation within the myosin motor domain: implications for structure and function. Structure. 1996;15:969-987.

42. Liu XZ, Walsh J, Mburu P, et al. Mutations in the myosin VIIA gene cause non-syndromic recessive deafness. Nat Genet. 1997;16:188190.

43. Ahmed ZM, Riazuddin S, Aye S, et al. Gene structure and mutant alleles of PCDH15: nonsyndromic deafness DFNB23 and type 1 Usher syndrome. Hum Genet. 2008;124:215-223.

44. Zheng QY, Yan D, Ouyang XM, et al. Digenic inheritance of deafness caused by mutations in the genes encoding cadherin 23 and protocadherin 15 in mice and humans. Hum Mol Genet. 2005;14:103-111.

45. Aller E, Jaijo T, Garcia-Garcia G, et al. Identification of large rearrangements of the PCDH15 gene by combined MLPA and a CGH: large duplications are responsible for Usher syndrome. Invest Ophthalmol Vis Sci. 2010;51:5480-5485. 